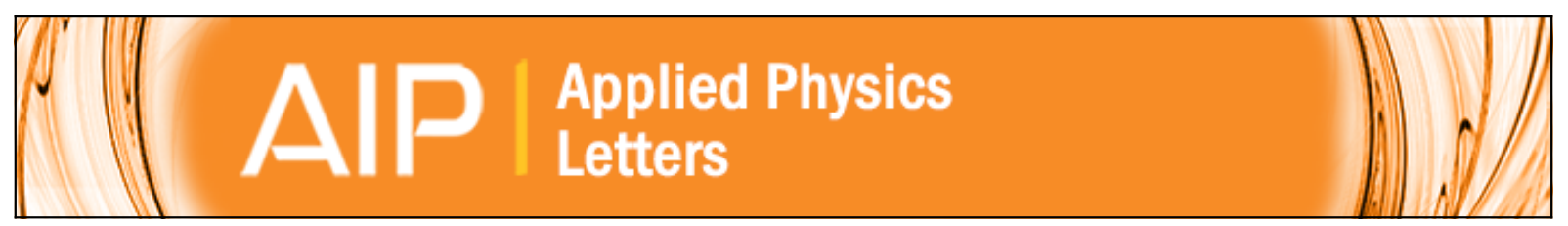

\title{
Wide angle terahertz sensing with a cross-dipole frequency selective surface
}

P. Rodríguez-Ulibarri, S. A. Kuznetsov, and M. Beruete

Citation: Applied Physics Letters 108, 111104 (2016); doi: 10.1063/1.4944326

View online: http://dx.doi.org/10.1063/1.4944326

View Table of Contents: http://scitation.aip.org/content/aip/journal/apl/108/11?ver=pdfcov

Published by the AIP Publishing

\section{Articles you may be interested in}

Optically transparent frequency selective surfaces on flexible thin plastic substrates

AIP Advances 5, 027107 (2015); 10.1063/1.4907929

Infrared transparent frequency selective surface based on metallic meshes

AIP Advances 4, 027112 (2014); 10.1063/1.4866292

Terahertz filters based on frequency selective surfaces for high-speed terahertz switch

J. Appl. Phys. 113, 014504 (2013); 10.1063/1.4773341

Ohmic loss in frequency-selective surfaces

J. Appl. Phys. 93, 5346 (2003); 10.1063/1.1565189

Fabrication of frequency-selective surfaces using microlens projection photolithography

Appl. Phys. Lett. 80, 3500 (2002); 10.1063/1.1477941

\section{Precise temperature control for cryogenic research}

Model 372

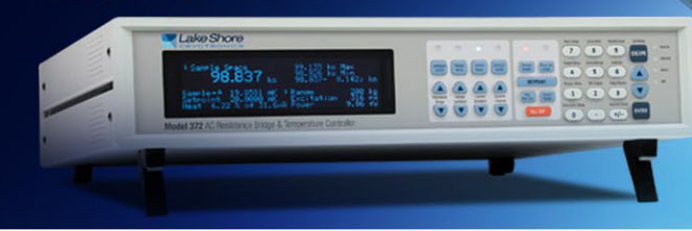




\title{
Wide angle terahertz sensing with a cross-dipole frequency selective surface
}

\author{
P. Rodríguez-Ulibarri, ${ }^{1, a)}$ S. A. Kuznetsov, ${ }^{2,3,4, b)}$ and M. Beruete ${ }^{1,5, c)}$ \\ ${ }^{1}$ Antennas Group-TERALAB, Universidad Pública de Navarra, 31006 Pamplona, Spain \\ ${ }^{2}$ Novosibirsk State University, ATIC HT\&NM, Pirogova Str. 2, 630090 Novosibirsk, Russian Federation \\ ${ }^{3}$ Budker Institute of Nuclear Physics SB RAS, Lavrentiev Ave. 11, 630090 Novosibirsk, Russian Federation \\ ${ }^{4}$ Institute of Semiconductor Physics SB RAS, Novosibirsk Branch "TDIAM," Lavrentiev Ave. 2/1, \\ 630090 Novosibirsk, Russian Federation \\ ${ }^{5}$ Institute of Smart Cities, Universidad Pública de Navarra, 31006 Pamplona, Spain
}

(Received 23 November 2015; accepted 3 March 2016; published online 16 March 2016)

\begin{abstract}
In this work, a terahertz sensor based on a cross dipole frequency selective surface is analyzed and experimentally tested. The sensing structure is optimized for operation at the fundamental bandstop resonance near $0.7 \mathrm{THz}$ and characterized under normal and oblique incidence. The sensing performance as a function of the incidence angle and the wave polarization is evaluated with good agreement between simulations and measurements. It is shown that a figure of merit for the proposed sensor can be enhanced from 0.2 up to 0.6 due to switching from normal to oblique excitation, which yields the maximum performance for TM polarization at the incidence angle of $70^{\circ}$. The presented results demonstrate a wide angle operation regime in $\mathrm{THz}$ sensing that opens up an alternative approach in improving capabilities of sensing devices. (C) 2016 AIP Publishing LLC.

[http://dx.doi.org/10.1063/1.4944326]
\end{abstract}

The terahertz $(\mathrm{THz})$ band $(0.1-10 \mathrm{THz})$ has been referred to as the "THz gap" for a long time, due to the lack of efficient generators and receivers operating at this frequency range. Although the development of components for a complete $\mathrm{THz}$ system is still troublesome, a great progress has been achieved by the scientific community over the last decade. ${ }^{1,2}$ Nowadays, many applications such as imaging, sensing, spectroscopy, and wireless communications have found their place in this spectral region. Given the location of the $\mathrm{THz}$ band between microwaves and infrared frequencies, a rich merging of concepts from microwave engineering, plasmonics, and optical disciplines can be applied. This endows the THz band with a marked interdisciplinary character that contributes to the continuous expansion of devices and applications.

Nowadays, sensing devices are widely present in numerous sectors such as security, fabrication process control, medicine, or communications, to name but a few. Thus, researchers have made a great effort in developing advanced sensing platforms, putting the emphasis in enhanced sensitivity, compactness and fabrication costs. In particular, plasmonics and metamaterials (MM) communities have focused their efforts on this field leading to promising devices. ${ }^{3}$

An important subgroup of infrared and optical sensing devices is based on the excitation of surface plasmon polaritons (SPP) at a metal-dielectric interface. Recently, a low frequency version, called "spoof plasmons," has been successfully proposed for THz sensing. ${ }^{4,5}$ In turn, classical frequency selective surfaces (FSSs) have been extended from microwaves to $\mathrm{THz}$ aiming at sensing applications. ${ }^{6,7}$ Therefore, a different paradigm has been opened by extending the typical application playground of FSSs from far-field operation (antenna radomes or spatial filters), to unexplored realms like sensing, which

\footnotetext{
${ }^{a)}$ Electronic mail: pablo.rodriguez@unavarra.es

${ }^{\text {b) }}$ Electronic mail: sakuznetsov@nsm.nsu.ru

c)Electronic mail: miguel.beruete@unavarra.es
}

primarily works in the near or even reactive-field. In this regard, classical FSSs employed in sensing applications, which are inspired by MM, are usually encompassed within the general denomination of metasurfaces. ${ }^{8-12}$

The key parameters of a sensor are its sensitivity and resonance linewidth. ${ }^{13}$ Usually the sensitivity, $S$, is given as a measure of the displacement of the resonance $\left(\Delta \lambda_{\text {res }}\right)$ with a variation of the refractive index unit (RIU) of the surrounding media $\left(S=\Delta \lambda_{\text {res }} / \Delta n\right)[\mu \mathrm{m} / \mathrm{RIU}]$. However, it can be also related with other parameters linked to the analyte such as thickness, volume, or concentration. ${ }^{14}$ In the present case, the thickness of the analyte should be taken into account when characterizing the sensor in terms of sensitivity. The resonance linewidth is related to the spectral resolution and is defined as the full-width at half-maximum (FWHM). In terms of the quality factor, Q, a small FWHM leads to high values of $\mathrm{Q}$ and vice versa. Therefore, to assess the performance of a sensor, a figure of merit $(F O M)$ relating sensitivity and resonance linewidth is usually employed: $F O M=S / W H M$. This means that, in practical terms, a large sensitivity and a narrow spectral width are the requirements for high performance sensors.

High-Q resonances can be achieved with asymmetric structures. ${ }^{10,15}$ However, a narrowband response can be also found in simpler FSS configurations under oblique incidence. In this work, a cross-dipole FSS operating at the $\mathrm{THz}$ band is presented. Its performance as an FSS-based sensor is numerically and experimentally evaluated. The structure is characterized under oblique incidence for both polarizations (TE and TM) of the impinging wave. Note that a similar structure has been previously proposed at optical wavelengths using gold nanocrosses designed for gas sensing applications. ${ }^{16}$ More recently, cross-dipole based absorbers have been analyzed as THz sensors. ${ }^{17}$

Figure 1 shows a schematic of the cross-dipole FSS unit cell and a picture of the fabricated sample along with its 


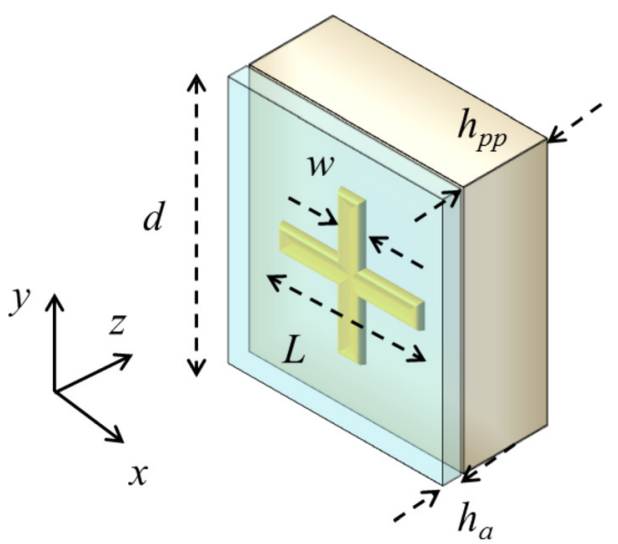

(a)

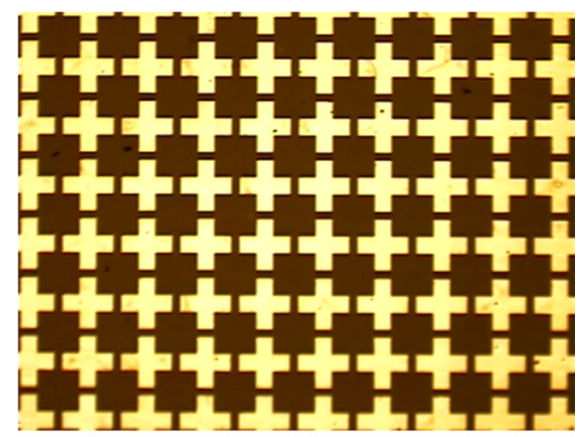

(b) physical dimensions. The prototype was fabricated on a thinfilm polypropylene (PP) substrate, which was chosen due to its low absorptivity and low dispersion in the $\mathrm{THz}$ band combined with good mechanical properties. ${ }^{18,19}$ The FSS was lithographically patterned in a $0.35 \mu \mathrm{m}$ thick aluminum (Al) layer sputtered on the PP film via a vacuum thermal deposition method. Prior to sputtering, the PP film was treated with a glow discharge in Ar atmosphere to improve adhesion of Al to PP. To pattern the FSS, we employed a contact photolithography technique, which was specifically adapted to flexible solid film substrates, such as PP, whose industrial production does not allow obtaining a liquid material suitable for posterior film deposition via spin coating. ${ }^{19}$ The sensing capability of the cross-dipole FSS was tested by depositing a thin layer on a photoresist material (AZ9260 from Microchemicals) that was spun coated over the fabricated sample, to serve as the analyte sensing body. Two different analyte thicknesses of 9 and $20 \mu \mathrm{m}$ were numerically and experimentally investigated. These values were determined after deposition by using a micrometer.
The structure shown in Fig. 1 was simulated using the frequency domain solver of the commercial electromagnetic software CST Microwave Studio ${ }^{\mathrm{TM}}$. To model the structure as an infinite array, the regime of Floquet ports and periodic boundary conditions applied to the FSS unit cell was employed. The complex permittivity of the PP film, real part and loss tangent, was extracted from the literature $^{18}\left(\varepsilon_{p p}=2.25, \tan \delta_{p p}=0.0005\right)$. For the photoresistive material (analyte), whose dielectric properties were initially unknown, the material parameters $\varepsilon_{a}=2.855$ and $\tan \delta_{a}$ $=0.05$ were retrieved from the experimental data by using a best fit to the simulations.

Figure 2(a) shows the transmission of the cross-dipole FSS without photoresistive material and when an analyte with thickness 9 and $20 \mu \mathrm{m}$ is deposited on top. The presented data demonstrate good agreement between simulations and measurements. A small discrepancy is explained mainly by inaccuracy of the analyte thickness determination $( \pm 0.2 \mu \mathrm{m})$, as well as by the thickness spread over the sample surface when depositing the analyte $( \pm 0.2 \mu \mathrm{m})$. The
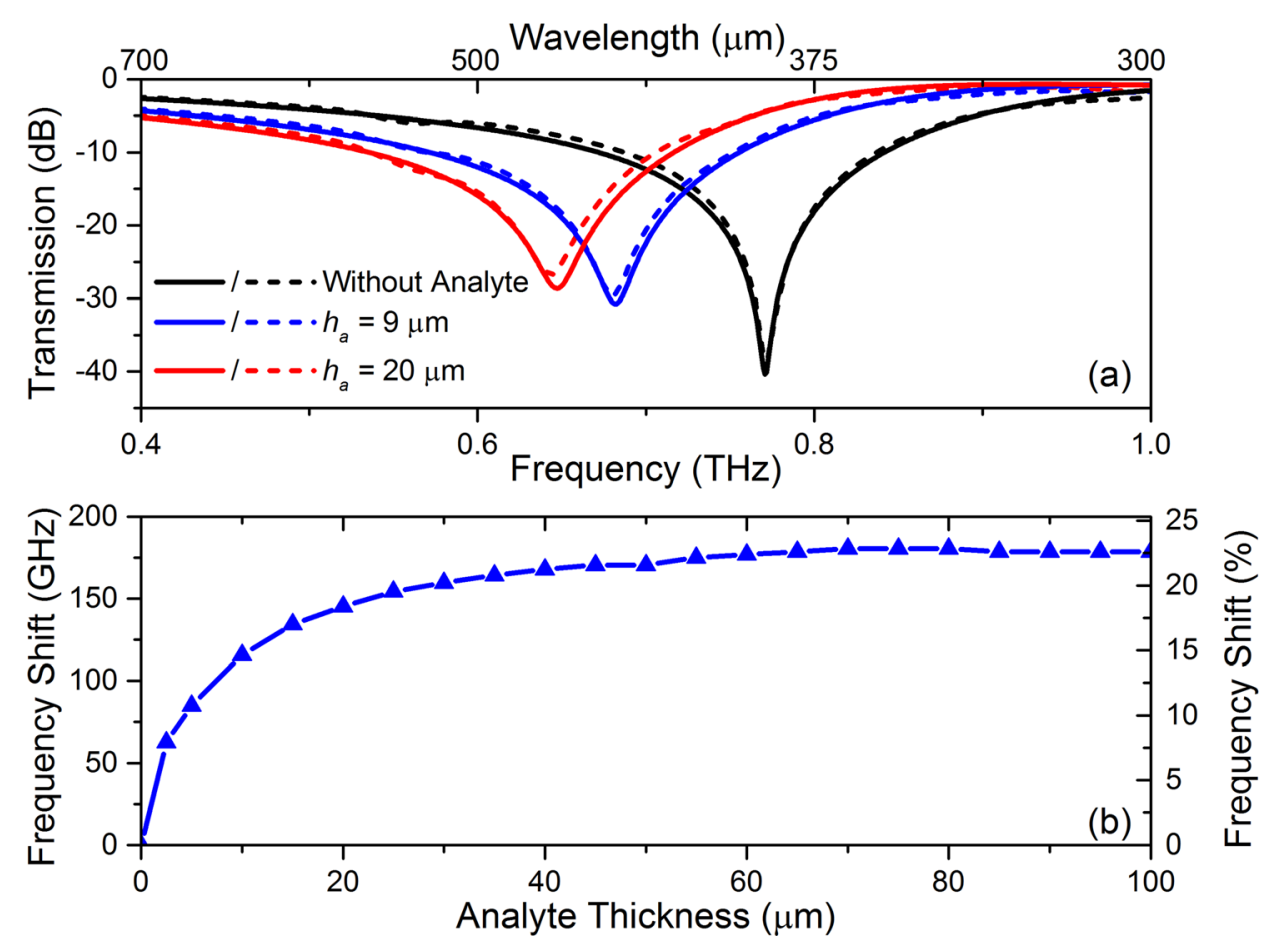

FIG. 2. (a) Transmission of the fabricated structure under normal incidence. Simulation (solid line) and measurement (dashed line) results for the analyte-free prototype (black curve), and after depositing an analyte with thicknesses of $h_{a}=9 \mu \mathrm{m}$ (blue curve) and $h_{a}=20 \mu \mathrm{m}$ (red curve). (b) Simulation results of the dipolar resonance frequency shift versus analyte thickness; (left axis) absolute frequency shift; (right axis) frequency shift normalized to the resonance frequency expressed in percentage. 
experimental measurements shown here were carried out with the TPS Spectra 3000 from Teraview. ${ }^{20}$ It can be seen that without the analyte, the cross-dipole FSS exhibits a stopband resonance positioned at $f_{\text {res }} \cong 770 \mathrm{GHz}$. This resonance represents the fundamental dipolar resonance of the metallic cross-dipole elements whose free-space wavelength $\lambda_{\text {res }}$ is primarily determined by the cross arm length $L$ and the effective permittivity $\varepsilon_{e f f}$ of the dielectric environment "seen" by the FSS: $\lambda_{\text {res }} \approx \chi \cdot L \cdot\left(\varepsilon_{e f f}\right)^{1 / 2}$. For the substrate thickness $h_{P P} \geq 0.1 \cdot \lambda_{\text {res }}$, which is satisfied in our case, the value of $\varepsilon_{e f f}$ is calculated as $\left.\varepsilon_{e f f}\right|_{h_{a}=0}=\left(1+\varepsilon_{P P}\right) / 2 \approx 1.63 .^{21,22}$ The parameter $\chi$ is known to be close to 2 for the cross-dipoles with narrow arms, ${ }^{21,22}$ though its exact value depends on the inter-cross spacing $(d-L)$ and the cross arm width $w^{23,24}$ yielding the actual quantity $\chi \approx 1.75$ for the dimensions, as indicated in Fig. 1(a). It should be highlighted that the presence of the analyte increases $\varepsilon_{e f f}$, inducing a redshift of the dipolar resonance. Moreover, when the analyte thickness $h_{a}<0.1 \cdot \lambda_{\text {res }}, \varepsilon_{e f f}$ appears to be a rapidly growing function of $h_{a}$ which asymptotically approaches the saturation level $\left.\varepsilon_{e f f}\right|_{h_{a} \rightarrow \infty}=\left(\varepsilon_{a}+\varepsilon_{P P}\right) / 2 \approx 2.55$. A fast change of $\varepsilon_{e f f}$ versus $h_{a}$ is of primary importance for sensors as it allows one to measure small values of $h_{a}$ by tracking a resonance frequency shift $\Delta f_{\text {res }}\left(h_{a}\right)=\left|\left(\left.f_{\text {res }}\right|_{h_{a} \neq 0}-\left.f_{\text {res }}\right|_{h_{a}=0}\right)\right|$. The behavior of $\Delta f_{\text {res }}\left(h_{a}\right)$ is illustrated in Figure 2(b) which shows that the frequency shift saturates for $h_{a}$ thicker than $60 \mu \mathrm{m}$, reaching a maximum value of about $180 \mathrm{GHz}$.

The FSS transmittance simulated under the condition of varying the real part of the analyte's complex refractive index between 1 and 2 with a step of 0.2 is shown in Figs. 3(a) and 3(b), which correspond to the analyte thicknesses $h_{a}=9$ and $20 \mu \mathrm{m}$, respectively. These results are summarized in Fig. 3(c) where the frequency shift of the dip resonance as a function of frequency is depicted. A maximum frequency shift of about $128.8 \mathrm{GHz}$ and $180 \mathrm{GHz}$ can be observed for $h_{a}=9$ and $20 \mu \mathrm{m}$, respectively. From these curves, we obtain that the sensitivity varies between 15 and $20 \%(68.7$ and $89 \mu \mathrm{m} / \mathrm{RIU})$ for $h_{a}=9 \mu \mathrm{m}$ and between 20 and 27\% (102.6 and $138.6 \mu \mathrm{m} / \mathrm{RIU}$ ) for $h_{a}=20 \mu \mathrm{m}$, see Fig. 3(d). Regarding the FoM, plotted in the same figure, the low $\mathrm{Q}$ nature of the resonance gives as a result the moderate values, ranging between [0.12-0.15] and [0.16-0.22] for $h_{a}=9 \mu \mathrm{m}$ and $20 \mu \mathrm{m}$, respectively [see Fig. 3(d)].

Next, the performance under oblique incidence was evaluated. As usual, the structure was characterized in the two principal polarization planes, TE and TM. Figures 4(a) and 4(b) show the sensitivity for an analyte thickness of $h_{a}=9 \mu \mathrm{m}$ under TM and TE illumination, respectively, with an incidence angle $\theta=45^{\circ}$. For TM polarization [Fig. 4(a)], the fundamental mode resonance only experiences a small blueshift of $16.2 \mathrm{GHz}$ with respect to the normal incidence case. It can also be noticed the appearance of two small resonances in the spectrum near the fundamental resonance dip (they take place at $838 \mathrm{GHz}$ and $877 \mathrm{GHz}$ for the case $\left.\operatorname{Re}\left\{n_{a}\right\}=1\right)$. The second resonance corresponds to the bent mode occurring at oblique incidence angles and for TM polarization, ${ }^{21,25,26}$ while the third resonance is related with the onset of the first grating lobe. The origin of the bent mode is the excitation of an asymmetrically induced current due to the coupling between the horizontal and vertical dipoles. ${ }^{25,26}$ The surface currents excited on the cross dipole for the fundamental and bent modes are displayed in Fig. 4(a) insets. On the contrary, for TE polarization the principal resonance only experiences a redshift of $94.5 \mathrm{GHz}$ that corresponds to approximately $10 \%$ of the resonant frequency. This dependence of the fundamental resonance with the angle of incidence under TE polarization is the classical behavior of cross-dipole and dipole FSS. ${ }^{25-28}$ This shift can be explained by means of equivalent circuit approaches, as it was done in Ref. 28. As the angle of incidence increases, the cut-off of the first higher-order TE harmonic also decreases and consequently the resonant frequency is redshifted. This feature permits using the sensor at different frequency ranges by just varying the angle of incidence.

In order to fully characterize the sensing performance under oblique incidence, CST simulations have been run wherein the incidence angle varied from 0 to $85^{\circ}$ with a step
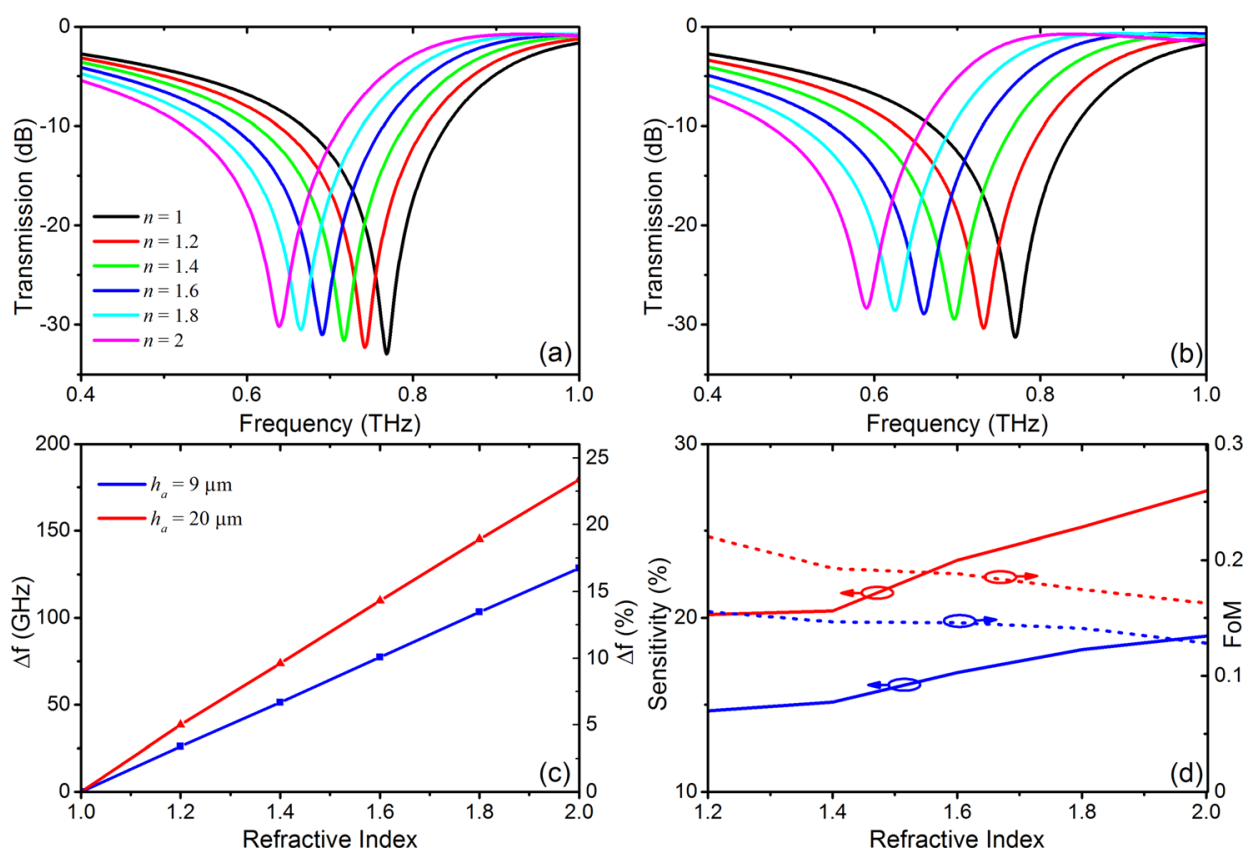

FIG. 3. (a) Simulated normal incidence transmission for the prototype with analyte thickness of $h_{a}=9 \mu \mathrm{m}$ at different refractive index values. (b) Same as (a) for $h_{a}=20 \mu \mathrm{m}$. (c) Frequency shift of the resonance vs. the variation of the refractive index. (d) Sensitivity and FoM. 

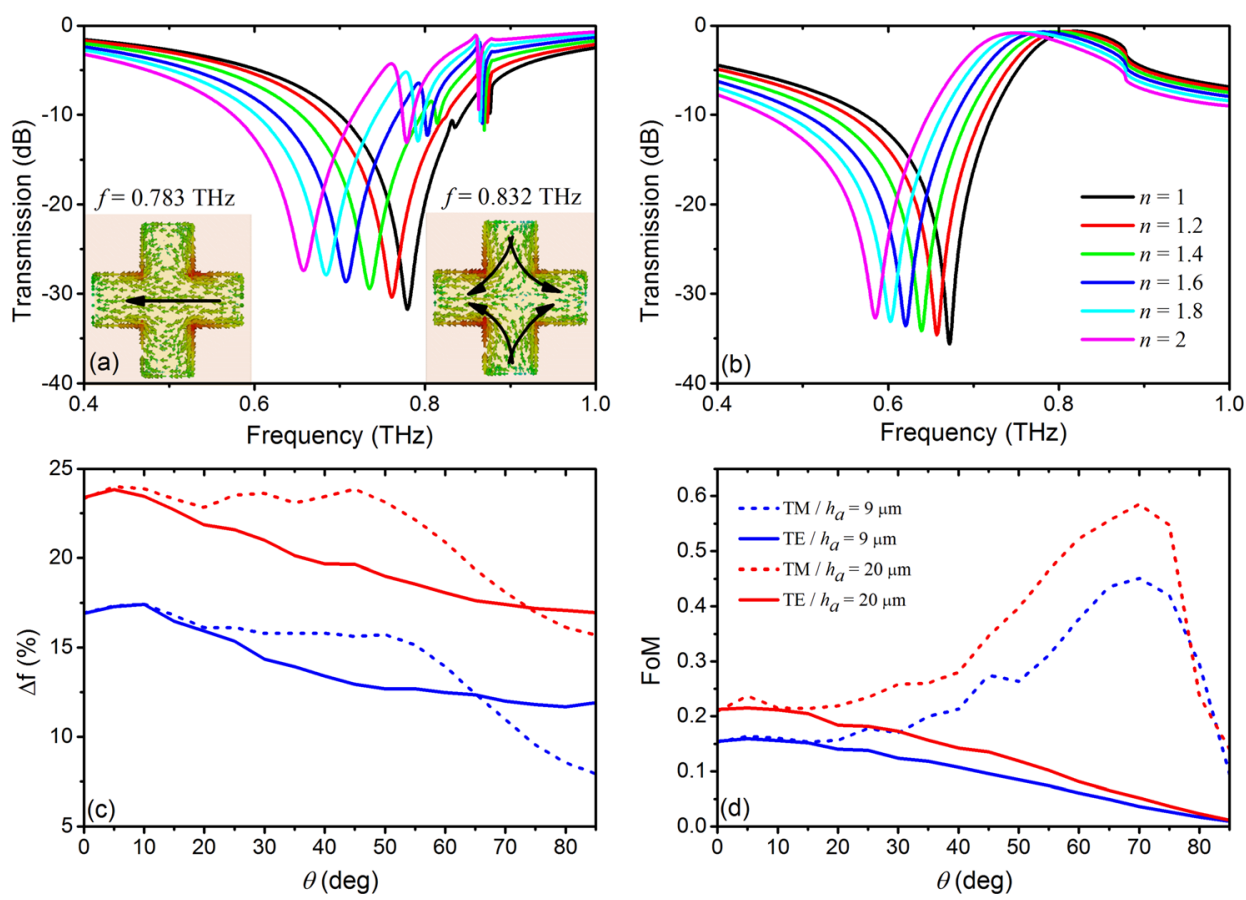

FIG. 4. (a) Simulated transmission results for the prototype with an analyte of thickness $h_{a}=9 \mu \mathrm{m}$ and $\theta=45^{\circ}$ (TM polarization) Insets: Simulated surface currents for the fundamental (left) and bend mode (right) resonances. (b) Same as (a) for TE incidence. (c) Maximum frequency shift vs. angle of incidence for $h_{a}=9$ and $20 \mu \mathrm{m}$ under TE (solid lines) and TM (dashed lines) excitation. (d) Maximum FoM vs. angle of incidence for $h_{a}=9$ and $20 \mu \mathrm{m}$ under TE (solid lines) and TM (dashed lines) excitation. of $5^{\circ}$. Figure $4(\mathrm{c})$ shows the maximum frequency shift of the dipolar resonance under both TE and TM polarizations. It can be observed that for the TM case a wide angle operation can be achieved with good sensitivity performance. Figure 4(d) shows the maximum FoM obtained versus the angle of incidence. These results reveal that a wide angle operation can be attained for TM excitation also in terms of FoM. In fact, the largest value is obtained near $70^{\circ}$, with a peak reaching a value around 0.6 , i.e., surpassing three times the case of normal incidence. In contrast, for TE polarization, the FoM linearly decreases with the angle of incidence. However, a fair performance is obtained within an angular width of $40^{\circ}$. Therefore, it can be stated that oblique incidence enhances the sensing performance of this device in several aspects. First, for the TM case, the higher $S$ and FoM values are obtained. Furthermore, this behavior occurs at slightly higher frequencies in comparison with the normal incidence case enlarging the operational frequency range. For TE, although the sensing performance is less competitive, a noticeable downshift in the fundamental resonance is experienced enlarging the operation range towards lower frequencies.

In order to corroborate the numerical results, an experimental characterization of the oblique response with a photoresist thickness of $20 \mu \mathrm{m}$ was carried out. To this end, an $\mathrm{ABmm}^{\mathrm{TM}}$ vector network analyzer along with a quasioptical bench was used. ${ }^{29}$ The angle of incidence $\theta$ was computer controlled using an angular positioner. The spectral measurements were performed within the band of $0.63-1 \mathrm{THz}$ with a frequency step of $200 \mathrm{MHz}$, while the angle $\theta$ varied from $0^{\circ}$ to $60^{\circ}$ with an increment of $5^{\circ}$. Measurements at larger incidence angles were not possible due to instrumental limitations, since beyond $60^{\circ}$ the sample holder obstructs largely the incidence beam. Figure 5 shows

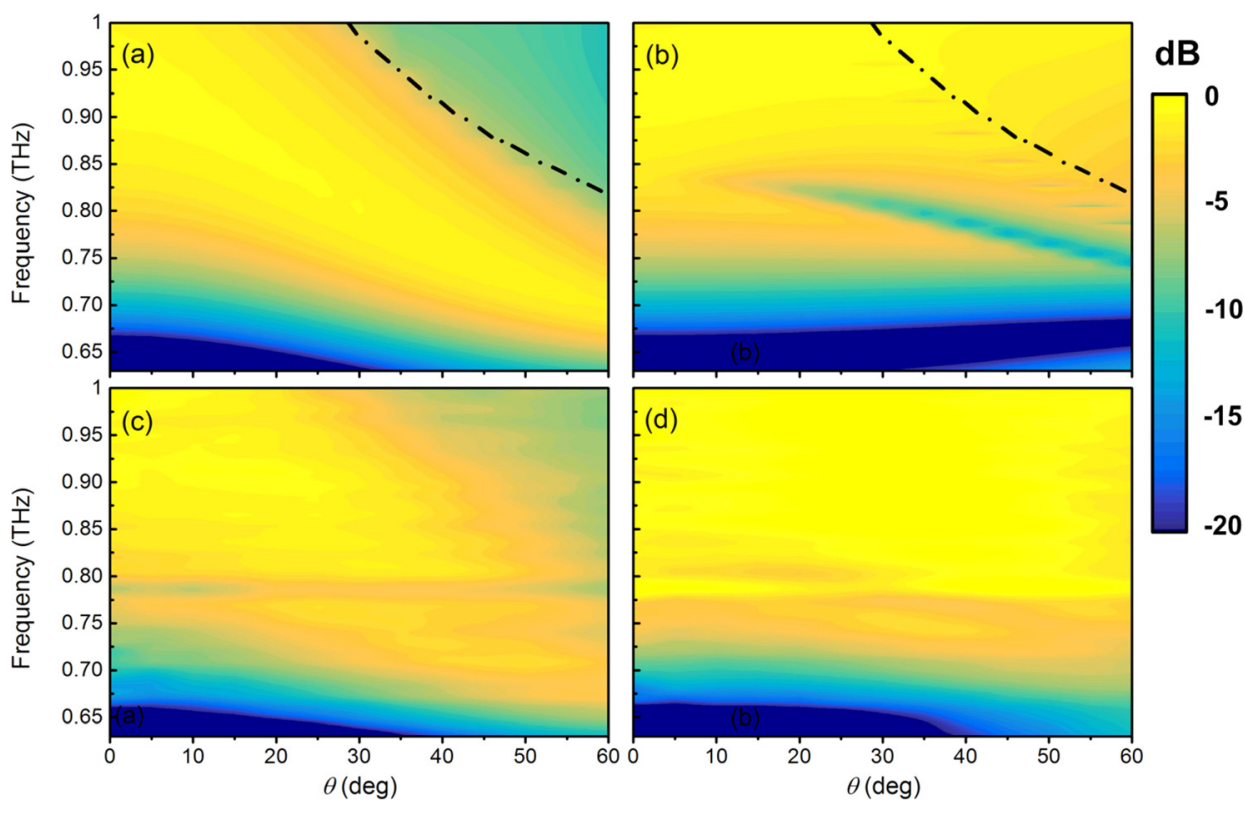

FIG. 5. Simulated and measured transmission for the prototype with an analyte of thickness $h_{a}=20 \mu \mathrm{m}$ with incidence angle $\theta$ varying from 0 to $60^{\circ}$. (a) TM, simulations. (b) TE, simulations. (c) TM, measurements. (d) TE, measurements. Dashed-dotted black contour line in (a) and (b) corresponds with the onset of the first order grating lobe. 
the comparison between CST Microwave Studio simulations and measurements for TM and TE polarization. In Figs. 5(a) and 5(b), the onset of the grating lobe is superimposed as a solid-black line. An overall good qualitative agreement is observed between simulations and experiments. Although less marked, the bent mode for TM polarization is also observed in the experiment (especially between $30^{\circ}$ and $40^{\circ}$ ). On the other hand, the small resonance just before the onset of the grating lobe vanishes in the experiment. For TE polarization, the agreement is also good. Only a small blueshift in frequency is identified experimentally as well as a small ripple coming from multiple reflections occurring in the set-up. The differences between measurements and simulations are mainly due to finite structure effects. What is more, in the experiment the structure is excited by a Gaussian beam instead of a pure plane wave. In addition, only a limited number of cross elements are illuminated and this number decreases as the angle of incidence increases.

In summary, a classical FSS has been demonstrated for terahertz sensing. It has been numerically and experimentally tested under normal incidence providing a polarizationindependent fair performance with FoM values of 0.2 . This value is enhanced up to 0.6 for oblique incidence for TM polarization. For TE polarization, the performance is deprecated. However, the redshift of the response permits sensing in the different frequency range enlarging the operational band of the device. Although in terms of FoM, other solutions could be more competitive ${ }^{16}$ the simplicity of our structure makes it a good candidate for $\mathrm{THz}$ sensing. Its design process can be readily conducted by using numerical simulators and even in a faster way by using analytical approaches based on transmission line networks for $2 \mathrm{D}$ periodic metallic screens embedded in layered media. ${ }^{30}$ Furthermore, the device is quite compact having a total thickness of $0.13 \lambda_{0}$ at $770 \mathrm{GHz}$.

This work was sponsored by the Spanish Government under Contract Nos. TEC2011-28664-C02-01 and TEC2014-51902-C2-2-R. P.R.-U. was sponsored by Public University of Navarra via a predoctoral scholarship. M.B. was sponsored by the Spanish Government via RYC-201108221. S.A.K. was supported by the Ministry of Education and Science of the Russian Federation under the State Assignment Contract \#3002.
${ }^{1}$ P. H. Siegel, IEEE Trans. Microwave Theory Tech. 50, 910 (2002).

${ }^{2}$ B. Ferguson and X. Zhang, Nat. Mater. 1, 26 (2002).

${ }^{3}$ T. Chen, S. Li, and H. Sun, Sensors 12, 2742 (2012).

${ }^{4}$ B. Ng, J. Wu, S. M. Hanham, A. I. Fernández-Domínguez, N. Klein, Y. F. Liew, M. B. H. Breese, M. Hong, and S. Maier, Adv. Opt. Mater. 1, 543 (2013).

${ }^{5}$ B. You, J.-Y. Lu, T.-A. Liu, and J.-L. Peng, Opt. Express 21, 21087 (2013).

${ }^{6}$ C. Debus and P. H. Bolivar, Appl. Phys. Lett. 91, 184102 (2007).

${ }^{7}$ R. Dickie, R. Cahill, V. Fusco, H. S. Gamble, and N. Mitchell, IEEE Trans. Terahertz Sci. Technol. 1, 450 (2011).

${ }^{8}$ T. Driscoll, G. O. Andreev, D. N. Basov, S. Palit, S. Y. Cho, N. M. Jokerst, and D. R. Smith, Appl. Phys. Lett. 91, 062511 (2007).

${ }^{9}$ B. Lahiri, A. Z. Khokhar, R. M. De La Rue, S. G. McMeekin, and N. P. Johnson, Opt. Express 17, 1107 (2009).

${ }^{10}$ R. Singh, W. Cao, I. Al-Naib, L. Cong, W. Withayachumnankul, and W. Zhang, Appl. Phys. Lett. 105, 171101 (2014).

${ }^{11}$ S. J. Park, J. T. Hong, S. J. Choi, H. S. Kim, W. K. Park, S. T. Han, J. Y. Park, S. Lee, D. S. Kim, and Y. H. Ahn, Sci. Rep. 4, 4988 (2014).

${ }^{12}$ H. Tao, L. R. Chieffo, M. A. Brenckle, S. M. Siebert, M. Liu, A. C. Strikwerda, K. Fan, D. L. Kaplan, X. Zhang, R. D. Averitt, and F. G. Omenetto, Adv. Mater. 23, 3197 (2011).

${ }^{13}$ W.-S. Tsai, K.-L. Lee, M.-Y. Pan, and P.-K. Wei, Opt. Lett. 38, 4962 (2013).

${ }^{14}$ J. F. O'Hara, W. Withayachumnankul, and I. Al-Naib, J. Infrared, Millimeter, Terahertz Waves 33, 245 (2012).

${ }^{15}$ C. Jansen, I. A. I. Al-Naib, N. Born, and M. Koch, Appl. Phys. Lett. 98, 051109 (2011).

${ }^{16}$ F. J. Rodríguez-Fortuño, M. Martínez-Marco, B. Tomás-Navarro, R. Ortuño, J. Martí, A. Martínez, and P. J. Rodríguez-Cantó, Appl. Phys. Lett. 98, 133118 (2011).

${ }^{17}$ L. Cong, S. Tan, R. Yahiaoui, F. Yan, W. Zhang, and R. Singh, Appl. Phys. Lett. 106, 031107 (2015).

${ }^{18}$ R. Ortuño, C. García-Meca, and A. Martínez, Plasmonics 9, 1143 (2014).

${ }^{19}$ M. Navarro-Cía, S. A. Kuznetsov, M. Aznabet, M. Beruete, F. Falcone, and M. Sorolla, IEEE J. Quantum Electron. 47, 375 (2011).

${ }^{20}$ See http://www.teraview.com for Teraview-Terahertz Products; accessed 9 February 2016.

${ }^{21}$ B. A. Munk, Frequency Selective Surfaces: Theory and Design (WileyInterscience, USA, 2000).

${ }^{22}$ M. E. MacDonald, A. Alexanian, R. A. York, Zoya Popović, and E. N. Grossman, IEEE Trans. Microwave Theory Tech. 48, 712 (2000).

${ }^{23}$ K. D. Moller, K. R. Farmer, D. V. P. Ivanov, O. Sternberg, K. P. Stewart, and P. Lalanne, Infrared Phys. Technol. 40, 475 (1999).

${ }^{24}$ C. T. Cunningham, Infrared Phys. 23, 207 (1983).

${ }^{25}$ E. Pelton and B. Munk, IEEE Trans. Antennas Propag. 27, 323 (1979).

${ }^{26}$ C. H. Tsao and R. Mittra, IEEE Trans. Antennas Propag. 32, 478 (1984).

${ }^{27}$ G. I. Kiani, K. P. Esselle, K. L. Ford, A. R. Weily, and C. Panagamuwa, Microwave Opt. Technol. Lett. 50, 2315 (2008).

${ }^{28}$ M. García-Vigueras, F. Mesa, F. Medina, R. Rodríguez-Berral, and J. L. Gómez-Tornero, IEEE Trans. Antennas Propag. 60, 4637 (2012).

${ }^{29}$ See http://www.abmillimetre.com for AB Millimetre-Company Specialized in MVNA manufacturing; accessed 9 February 2016.

${ }^{30}$ R. Rodríguez-Berral, F. Mesa, and F. Medina, IEEE Trans. Microwave Theory Tech. 63, 1969 (2015). 